\title{
Recambio parcial con solución salina en el recién nacido poliglobúlico
}

\author{
Pablo Valenzuela F. ${ }^{1}$; María Eugenia Hubner G. ${ }^{1}$; \\ Pilar Lenero M.2; Elizabeth Araya L. 2; Ana María González A.2; \\ Maris Zambrano S.2; Elisa Standen V.2
}

\section{Exchange of blood for sodium chloride solution in neonatal polycythemia}

Exchange of blood for plasmo or saline i $\mathrm{NaCl} 0.9 \%$ in water solution were done respectively in two groups in: 12 eachl of polycythemic newborn infanis, to decrease their hemalociit values toward $55 \%$ and to sludy the effect of lhe $\mathrm{NaCl}$ solution on palients hemodinamics and acid-base vaisables. Peripheral veins and arteries were used in all cases for infusion of fluids and blood withdrawal. The desired hemalociit was aftained in all palients and no slatlstically signilicani differences were lound regarding to linal hematocril, and serum $\mathrm{Na}, \mathrm{Cl}, \mathrm{K}$, proleins, glucose and bilirubin cancentration neilher blood $\mathrm{pH}$ variations and no cases were recorded in which changes in these crileria exeeded their normal ronges for newborn infants. Partial exchange of blood for $\mathrm{NaClO} 0.9 \%$ solution in water seems to be a safe procedure, potentially free of the risks of blood transmiled infections and the claims of some religious objectors.

Key words: polycythemia neonatal, exchange fransfusion, soline solution, sodium chloride, watereleclrolyte boance, serum protein level.

La poliglobulia ${ }^{1}$ es frecuente en todas las unidades de recién nacidos y su tratamiento usualmente consiste en cambiar la sangre por plasma a fin de disminuir el hemalocrito. Los riesgos que implica la transfusión de hemoderivados para el recién nacido han impulsado la búsqueda de otras opciones que reemplacen esos productos por soluciones artificiales, entre las que destaca -por su bajo coste y facilidad de manejo- Ja solución de $\mathrm{NaCl}$ al $0,9 \%$ en agua.

Además de la posibilidad de infección, al recambiar sangre por plasma, el recién nacido recibe una mezcla que difiere del plasma normal en cuanto a su composición en electrólitos y proteínas, pues $13,3 \%$ del líquido aportado está constiluido por solución de anticoagulante. De hecho, en nuestro hospital, corrientemente se mezclan un promedio de $525 \mathrm{ml}$ de sangre con $70 \mathrm{ml}$ del anticoagulante CPD (citrato, ácido cítrico, bifos-

1. Unidad de Neonalologla, Hospital Clínico Universidad de Chile.

2. Matrona. Unidad de Neonatologia, Hospita! Clínico Universidad de Chile. fato de sodio y dextrosa), cuyos componentes altamente metabolizables desaparecerán del espacio vascular rápidamente, quedando sólo agua a cambio. Esto implica dilución de las proteínas del plasma y aporte de agua pura. De cualquier modo este aporte puede parecer insignificante para un recambio de $15 \mathrm{ml}$ por $\mathrm{kg}(3,75 \mathrm{ml}$ por $\mathrm{kg}$ de agua pura). Asimismo, el recambio de sangre por plasma tendería a producir elevación de la concentración sérica de $\mathrm{Na}$ y $\mathrm{Cl}$ y descenso de las proteínas plasmáticas. Con respecto a la volemia, la hipernatremia y la hipoproteinemia tendrían efectos opuestos. Por otra parte, el recambio con solución de $\mathrm{NaCl} 0,9 \%$ significa aportes de $\mathrm{Na}$ y $\mathrm{Cl}$ levemente superiores a los extraídos y descenso de las proteínas. En la tabla 1 se describe un balance hipotético de $\mathrm{Na}, \mathrm{Cl}$ y proteinas en un caso de recambio con plasma y otro con solución $\mathrm{NaCl} 0,9 \%$.

El objetivo de este estudio fue describir los efectos del recambio parcial con solución saljna sobre el hematocrito, bilirrubinemia, sodio, potasio, cloro y proteinas plasmáticas en relación a los producidos por el recambio parcial con plasma. 


\section{Tabla 1}

Balance de $\mathrm{Na}, \mathrm{Cl}$ y proteínas en recambio parcial de sangre por plasma o solución de $\mathrm{NaCl} 0,9 \%$ en un recién nacido de $3000 \mathrm{~g}$ con hematocrito $70 \%$, volemia estimada de $88 \mathrm{ml} / \mathrm{kg}$ y hematocrito final deseado 60\%. Las cantidades extraídas o aportadas se expresan por $\mathrm{kg}$ de peso del recién nacido

\begin{tabular}{lccccc}
\hline & Extraído & Plasma & Balance & NaCl 0,9\% & Dalance \\
\hline Na mEq & 0,56 & 2,23 & $+1,67$ & 1,89 & $+1,33$ \\
Cl mEq & 0,41 & 1,00 & $+0,59$ & 1,89 & $+1,48$ \\
Proteinas $(\mathrm{g})$ & 0,22 & 0,60 & $+0,38$ & 0,0 & $-0,22$
\end{tabular}

\section{Tabla 2}

Datos generales de 24 recién nacidos poliglobúlicos sometidos a recambio parcial de sangre por plasma o solución $\mathrm{NaCl} 0.9 \%$

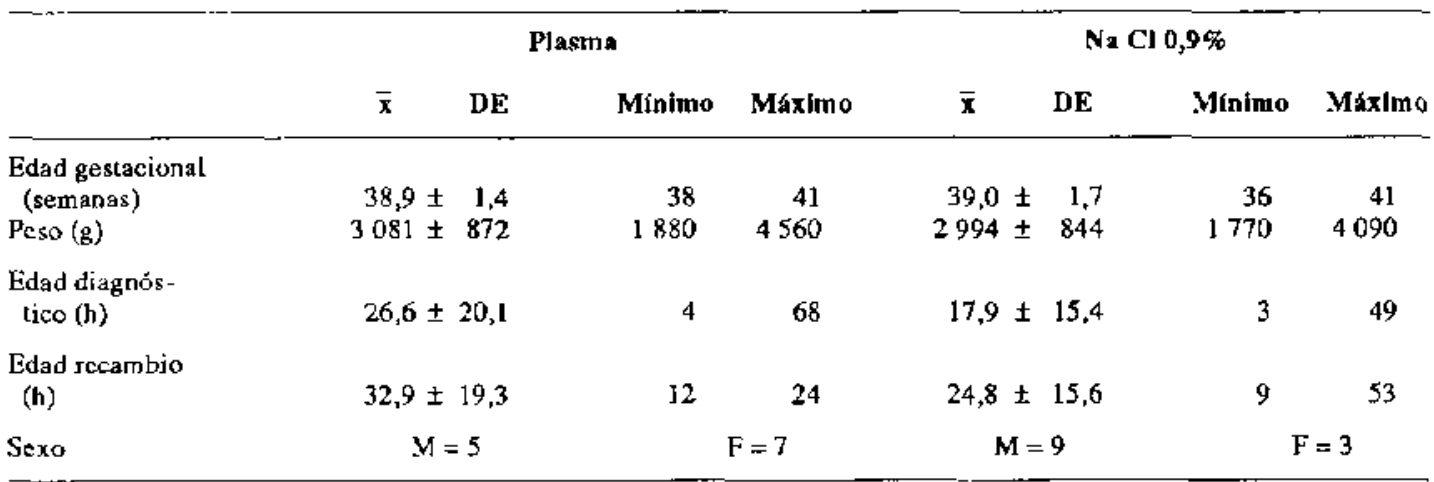

\section{Material y Métodos}

Se consideraron poliglobúlicos los recién nacidos cuyo hematocrito era $\geq 65 \%$. Ellos fueron sornetidos a recambio si el hemalocrito era $\geq 70 \%$, presentacen o no síntomas de hipcrviscosidad sanguínea o si estaba entre 65 y $70 \%$ cuando había síntomas atribuibles a hiperviscosidad2-4. Se excluycron de la experiencia los recién nacidos con cardiopatúas congénitas, insufjciencia renal, síndrome edematoso y los de pretémino con pesos inferiores a $1500 \mathrm{~g}$, sospecha clínica fundada de síndrome de dificulcad respiratoria idiopática o encefalopatia hipóxico isquémica. Los pacientes se asignaron al azar en la medida de la ocurrencia del diagnóstico, de modo que se altemó una u otræ forma paciente por medio. Los casos impares recibieron solución salina y los pares plasma. La asignación del primer recién nacjdo se decidió al azar. Doce recién nacidos fueron sontetidos a recambio de sangre por plasma $y$ un númeto igual con solución salina de $\mathrm{NaCl}$ al $0.9 \%$. El volumen a recambiar se estimó mediante la fómula de Uski y el monograma de Rawlings, teniendo como meta hematocrito $55 \% \%^{5}$. En $10^{-}$ dos los casos el procedirniento se realiź́ por via periférica, con sangrado arterial e infusion venosa?. Los recambios se realizaron en la Lnidad de Neonatología bajo condiciones de temperatura y humedad habiruales cn estos recintos. La frecuencia carbiaca y la temperatura se controlaron antes, durante y después del procedimiento. El seguimiento clínico consistió en contsol de los signos vitales y hemato. crito antes, inmediatamente después y al cabo de $24 \mathrm{~h}$ de realizado el procedimiento. Además se controlaron bilirrobina sériea, glicemia; sodio, cloro y potasio plasmáticos: pll y gases en sangre ancrial y proteinemia previa al rccambio y 24 h después de éste. Los valores obtenidos se expresaron en media y desviaciones estándar y e] significado de las diferencias se analizó por medio de la prueba t de Student, considerándose significativos valores de $\mathrm{p}$ infe. riores a 0,05 .

\section{Resultados}

En la tabla 2 se describen los datos biofísicos de los pacientes y el momento en que se realizó el recambio. Ambos grupos difieren sólo en la distribucion del sexo y la edad en que se efectuó el diagnóstico y el procedimiento. El tiempo entre ambos eventos fue similar para los dos grupos. 
En las tablas 3 y 4 se describen las variaciones que experimentaron los parámetros observados inmediatamente después y al cabo de 24 horas de realizado ei recambio con plasma o con solución de $\mathrm{NaCl} 0,9 \%$. Se puede observar que en ambos grupos se consiguió el objetivo de bajar el hematocrito a niveles deseados, con alto grado de cumplimiento en cuanto al recambio ideal, siendo éste un poco superior para el grupo de plasma que el salino, manteniéndose en ambos casos, a las $24 \mathrm{~h}$, en niveles previstos de $55,7 \%$ y $57 \%$, respectivamente ( $\mathrm{p}$ : $\mathrm{ns}$ ). En la concentración plasmática de electrólitos no se detcctaron variaciones significativas de $\mathrm{Na}, \mathrm{K}$ y $\mathrm{Cl}$ dentro de cada grupo y entre ellos. La bilirrubinemia experimentó una leve variación, que no fue significativa, sino más bien parece la propia de la evolución normal de ella entre el segundo y cuarto día de vida. No parece haber influencia del hematocrito sobre la bilirubinemia de este grupo. El grupo sometido a recambio salino experimentó una pequeña baja en la proteinemia, pero ella no fue estadísticamente significativa. Tampoco se registraron modificaciones significativas en el sanguíneo y lo mismo sucedió para el bicarbonato. Ningún caso mostró alteraciones del $\mathrm{PaCO}_{2}$. La glicemia no mostró variaciones significativas, tampoco la frecuencia cardíaca y la temperatura antes, durante y después del procedimiento.

\section{Comentario}

La poliglobulia es una entidad clínica ampliamente estudiada y de la cual se dispone de nutrida bibliografía sobre sus aspectos clínicos y secuelas, los que no son atingentes al motivo de esta prescntación. Hay escasa información sobre el uso de soluciones alternativas al plasma u otros derivados sanguíneos. El recambio de glóbulos rojos por plasma es una técnica relativamente reciente ${ }^{\beta}$ y en la actualidad otros hemodc-

Tabla 3

Variaciones de los parámetros estudiados frente al recambio parcial de sangre por plasma o solución de $\mathrm{NaCl} 0,9 \%$. Las diferencias no son significativas.

\begin{tabular}{|c|c|c|c|c|c|c|c|c|}
\hline & \multicolumn{2}{|c|}{ Hematocrito (\%) } & \multicolumn{2}{|c|}{ Sodto (mEqh) } & \multicolumn{2}{|c|}{ Potasio (mEq/I) } & \multicolumn{2}{|c|}{ Cloro $(\mathrm{mEg} / \mathrm{T})$} \\
\hline & Plasma & NaCl $0,9 \%$ & Plasma & $\mathrm{NaCl} 0,9 \%$ & Plasma & $\mathrm{NaCl} 0,9 \%$ & Plasma & $\mathrm{NaCl} 0,9 \%$ \\
\hline Prerrecambio & $70,0 \pm 3,6$ & $70,4 \pm 2,4$ & $139 \pm 6.4$ & $139 \pm 5,5$ & $4,8 \pm 1,0$ & $4,7 \pm 1,0$ & $108 \pm 10,1$ & $108 \pm 8,7$ \\
\hline Postrecambio & $53,5 \pm 4,5$ & $60,0 \pm 2,6$ & & & & & & \\
\hline 24 h despues & $55,7 \pm 4,9$ & $57,0 \pm 2,7$ & $138 \pm 4,1$ & $137 \pm 7.9$ & $4,0 \pm 0,9$ & $4,5 \pm 0,6$ & $98 \pm 8,0$ & $105 \pm 7,9$ \\
\hline
\end{tabular}

Los valores se expresan como promedio y DE.

Tabla 4

Variaciones de los parámetros estudiados frente al recambio parcial de sangre por plasma o solución de $\mathrm{NaCl} 0,9 \%$. Las diferencias no son significativas.

\begin{tabular}{|c|c|c|c|c|c|c|c|c|}
\hline & \multicolumn{2}{|c|}{ Billrrubina (mg \%) } & \multicolumn{2}{|c|}{ Proteinemia (g/l) } & \multicolumn{2}{|c|}{ Glicernia (mg \%) } & \multicolumn{2}{|c|}{$\mathrm{pH}$} \\
\hline & Plasma & NaCl 0,9\% & Plasma & $\mathrm{NaCl} 0,9 \%$ & Plasma & $\mathrm{NaCl} 0,9 \%$ & Plasma & $\mathrm{NaCl} 0,9 \%$ \\
\hline Prerrecambio & $7,5 \pm 3,3$ & $8,6 \pm 4,4$ & $6,0 \pm 1,4$ & $6,0 \pm 1,5$ & $49 \pm 13,2$ & $52 \pm 19$ & $7,43 \pm 0,05$ & $7,40 \pm 0,05$ \\
\hline Postrecambio & $6,5 \pm 2,8$ & $7,8 \pm 3,9$ & & & & & & \\
\hline 24 h después & $7,8 \pm 3,1$ & $10,0 \pm 2,9$ & $6,1 \pm 1,5$ & $5,1 \pm 1,0$ & $56 \pm 18,7$ & $52 \pm 11$ & $7,43 \pm 0,07$ & $7,42 \pm 0,06$ \\
\hline
\end{tabular}

Los valores se expresan como promedio y $\mathrm{DE}$. 
rivados lo han reemplazado 9 . Sin embargo, estos productos sanguíneos son caros, ocasionalmente difíciles de conseguir y no siempre exentos de riesgo de infecciones. Aún más, hay evidencias que sugieren que cl uso de plasma de adultos podria aumentar la viscosidad sanguínea del recién nacido ${ }^{10}$. Por otra parte, es sabido que hay grupos rcligiosos que rechazan el cmpleo dc hemoderivados, quedando los médicos en una difícil sítuación frente a estos recién nacidos po. liglobúlicos.

El uso de solución de $\mathrm{NaCl} 0,9 \%$ para producir hemodilución en el recién nacido no es un procedimiento nuevo, pero existen pocas referencias a evaluaciones de su uso. La presente investigación, aun cuando fue realizada con un número pequeño de recién nacidos, muestra resullados tan consistentes que hace aconsejable dejar definitivamente de lado el plasma en estos procedimientos, salvo que exista una indicación perentoria para su cmpleo. El uso sistemático de vías perifericas para el procedimiento ha significado una gran reducción en los costos y potencial perjuicio al recién nacido ${ }^{11}$.

\section{Resumen}

En 24 recién nacidos con poliglobulia se realizó de manera alternativa reemplazo de sangrc por plasma o por solución de $\mathrm{NaCl} 0,9 \%$, respectivamente, con el objeto de reducir el hematocrito a $55 \%$ y estudiar el efecto de la solución de $\mathrm{NaCl}$ sobre el agua, elcctrólitos, ácjdos, bases, proteínas, bilimubina, glucosa y $\mathrm{pH}$ en el sucro de los pacientes. En todos los casos se utilizaron vías venosas y arteriales periféricas. En ambos grupos se consiguió el objelivo planteado no encontrándose diferencias estadísticamente significativas en cuanto al hematocrito alcanzado y las restantes variables estudiadas en ambos sub- grupos, sin que tampoco en ellos se detectaran cambios que excediesen de los límites considerados como normales para la edad. El reemplazo parcial de sangre por solución de $\mathrm{NaCl} 0,9 \%$ es un procedimiento seguro, libre de infecciones o de impedimentos de tipo religioso.

(Palabras clave: policitemia neonatal, transfusión de recambio, solución salina, cloruto de sodio, balance hídrico, electrólitos, proteinemia.)

\section{Referencias}

1. Rivera $W$, Sanchez I, Cavagmaro $F$, Winter A: Poliglobulia Neonatal. Revisión de 110 casos. Rev Chil Pediatr 1988; 59: 257-260.

2. Wiswell TE, Cornish JD, Northam RS: Policitèmia neonatal: frecuencia de las manfestaciones clínicas y otros hallazgos asociados. Pediatrics (Ed. Esp) 1986; 22: 5-8.

3. Groos GP. Haihaway WE, McGaughey HR: Neonatal Hipperviscosity in the neonate. I Pediatr 1973; 82: 1004-1012.

4. Wirth FH, Goldberg KE, Lubchenco LO: Neonatal hiperviscosily: I. Incidence. Pediatrics 1979; 63; 833-836.

5. Oski FA and Naiman JL: Hernatologic problems in the ncwbom. Philadelphia, 1972, WB Saunders Co.

6. Rawling IS, Pettett G, Wiswell TE: Estimated blood volumes in polycythemics neonates as a function of birth weigh. J Pediatr 1982; 101: 594-599.

7. Valenzueía P, Zniorik $O$, Standen E: Recambio parcial por vía periférica en el recién nacido poliglobúlico. Trabajo presentado a las XIV Jomadas Chilenas de Pedialria, para publicación en Pediatría.

8. Wood $J$ : Phletora in the newborm infant associated with cyanosis and convulsions. I Pediatr 1959; 54: 1433.

9. Levy I, Meriob P, Ashkenazi S, and Reisner S: Neonatal polycythacmia: effect of partial dilutional exchange transfusion with human albumin on whow] blood viscosily. Eur J Pediatr 1990: 149: 354-355.

10. Linderkamp $O$, Versmold $H$, Riegel $K$, Betke $K$ : Contributions of red cells and plasma to blood hippervis. cosity in preterm and fullterm infants and adulis. Pediatrics 1984; 74: 45-51.

11. Black V, Rumack C, Lubchenco L, Koops B: Gastrointestintal injury in polycythemic tem infants. Pe diatrics $1985: 76$ : 225-231. 